\title{
Kinetic approach to the determination of the phase diagram of a solid solution
}

\author{
Marija Matovic $^{\mathrm{a}, *}$, J.C. van Miltenburg ${ }^{\mathrm{a}}$, H.A.J. Oonk ${ }^{\mathrm{a}}$, Jan H. Los ${ }^{\mathrm{b}}$ \\ ${ }^{a}$ Chemical Thermodynamics Group, University of Utrecht, Padualaan 8, 3584 CH Utrecht, The Netherlands \\ ${ }^{\mathrm{b}}$ IMM Laboratory of Solid State Chemistry, Radboud University Nijmegen, Toernooiveld, 6525 ED Nijmegen, The Netherlands
}

Received 13 September 2005; received in revised form 24 November 2005; accepted 24 November 2005

Available online 21 December 2005

\begin{abstract}
A binary mixture of 1,4-dichlorobenzene and 1,4-dibromobenzene, as a representative of a system that forms a solid solution, was measured in an adiabatic calorimeter. Several mixtures of different compositions were heated to $380 \mathrm{~K}$, kept in the liquid phase at this temperature overnight, and then cooled slowly down to $250 \mathrm{~K}$. The enthalpy path of the mixture measured during cooling carries the information that enables us to describe the crystallization process. In the first stage, crystallization develops rapidly and clearly deviates from equilibrium, while in the second stage it proceeds significantly slower, whereby we assume that the surface of the solid is in equilibrium with the existing liquid phase at the given temperature during cooling. Such an approach opposes the one that assumes equilibrium between the liquid and solid bulks, known as the equilibrium model. We show that by employing the kinetic model, the experimental enthalpy path of the mixture during cooling in the adiabatic calorimeter can be very successfully reproduced, while the equilibrium model fails in this aspect. Furthermore, we propose a procedure where the kinetic model is used to obtain the excess thermodynamic properties of the solid phase. These quantities enable the calculation of the liquid-solid phase diagram using the excess parameters obtained from the approach that does not assume the overall equilibrium between the phases during the crystallization process.
\end{abstract}

(c) 2005 Elsevier Ltd. All rights reserved.

\section{Introduction}

Multi-component systems of substances that have about the same size and shape of molecules and do not differ too much in chemical nature tend to form mixed crystals or solid solutions. Prediction of the phase behavior of such a mixture and characterization of the solid state require the knowledge of the relevant phase diagram. Several studies on the binary mixture of our interest, 1,4-dichlorobenzene and 1,4-dibromobenzene, demonstrate methods for determination of the equilibrium phase diagram [1-4]. Measuring equilibrium solidus and liquidus lines by melting samples is a laborious work in the sense of preparing mixtures of a high degree of homogeneity [5-7], while there is always uncertainty about whether the system has reached the equilibrium during measurements. As for the melting and crystallization of the molecular mixed crystals, the problem arises due to very low diffusion rates in the solid phase [8] that prevent overall equilibrium between the entire amounts of solid and

\footnotetext{
* Corresponding author. Tel.: +31 644298363.

E-mail address: m.matovic@chem.uu.nl (M. Matovic).
}

liquid phase. Therefore, the result of crystallization will be an inhomogeneous state of solid that contains composition gradients in its bulk. As an example, previous analysis of the mixture of 1,4-dichlorobenzene and 1,4-dibromobenzene by Raman spectroscopy illustrates the distribution of the given components along the length and the diameter of the single crystal [9].

In this work, we focus on the crystallization of the issued mixture in an adiabatic calorimeter, whereby we develop a kinetic model describing quantitatively the crystallization process. The basic assumption of the kinetic model is that at a slow cooling rate equilibrium is established between the surface of the growing solid phase and the existing liquid phase along the cooling path. Clearly, this is only valid for low enough cooling rates and thus for slow solidification processes. Nevertheless, no matter how slow the cooling is, due to the very slow diffusion rate in the solid phase, the final state of solid will not be the equilibrium state within a reasonable time scale, as we will show here.

For the purpose of modeling the slow crystallization close to equilibrium as performed in the adiabatic calorimeter, the 
effects of mass and heat transport in the liquid phase on the segregation can be excluded, as was demonstrated in Ref. [10]. Furthermore, it is shown how the applicability of the proposed kinetic model can be extended to determine the excess properties of the solid phase. In previous work [11], a preliminary description of this kinetic approach was given. Here, an improved model, that gives both excess enthalpy and excess entropy of the solid phase is presented in detail. Typically, a mixture with high miscibility of components exhibits lower excess energy and will form a solid solution, while for an eutectic mixture miscibility in the solid phase is limited corresponding to higher excess energy. As discussed in Ref. [12], the kinetics usually favors mixing. The advantage of proposed kinetic modeling over the traditional equilibrium approach [5] for the determination of excess properties is that it is not based on the unjustified assumption that the system is in complete equilibrium.

\section{Experimental method}

Several mixtures of 1,4-dichlorobenzene and 1,4-dibromobenzene of different compositions were measured in the adiabatic calorimeter (laboratory design indication CAL VII) [13]. Each mixture weighed around $5 \mathrm{~g}$ and was placed in a copper gold-plated vessel that was mounted in the calorimeter. The mixture was first heated slowly to $380 \mathrm{~K}$ in the way that was previously described [14], while the change of the enthalpy of the mixture with temperature is measured during the performed experimental procedure. Afterwards, the melt was kept overnight at $380 \mathrm{~K}$ and then cooled down to $250 \mathrm{~K}$ with a rate of $0.1 \mathrm{~K} \mathrm{~min}^{-1}$. Cooling in the adiabatic conditions is performed by setting the temperatures of the shields to relevant values with respect to the vessel temperature (see Fig. 1). Here by adiabatic conditions we mean that the applied cooling is the only heat exchange between the system, i.e. vessel and mixture, and its surroundings, i.e. shields and wire-heater. Thus, the enthalpy change of the system during cooling, $\mathrm{d} H_{\text {system }}$, within a time interval $\mathrm{d} t$ as a consequence of the cooling power or heat flow $I_{\text {cool }}=\mathrm{d} Q_{\text {cool }} / \mathrm{d} t$, with $Q_{\text {cool }}$ the withdrawn heat, is given by:

$\mathrm{d} H_{\text {system }}=\mathrm{d} H_{\text {ves }}+\mathrm{d} H_{\text {mix }}=\left(c_{p, \text { ves }}+c_{p, \text { mix }}\right) \mathrm{d} T=I_{\text {cool }} \mathrm{d} t$

where $\mathrm{d} H_{\text {ves }}$ and $\mathrm{d} H_{\text {mix }}$ are the enthalpy changes and $c_{p \text {,ves }}$ and $c_{p \text {,mix }}$ are the heat capacities of the vessel and the mixture, respectively.

During the cooling experiment the change of system's temperature with time, $\mathrm{d} T / \mathrm{d} t$, is measured, while the heat capacity of the empty vessel $c_{p \text {, ves }}$ as a function of temperature was measured independently in a calibration experiment. From the measured heat capacities of the mixtures in the one-phase temperature regions, we found only small fluctuating deviations from the ideal values, suggesting no significant excess heat capacity. This implies that the heat capacity of the mixture in the phase $P$ (liquid or solid phase) is given by:

$c_{p, \text { mix }}^{P}=(1-z) c_{p, 1}^{P}+z c_{p, 2}^{P}$

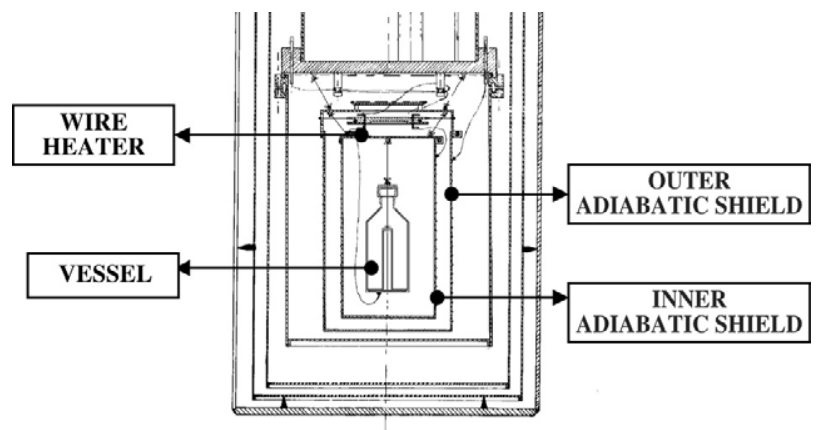

Fig. 1. The sketch of the sample container in the adiabatic calorimeter.

where $z=z_{2}$ is the overall composition of component $2(1,4-$ dibromobenzene), while $c_{p, 1}^{P}$ and $c_{p, 2}^{P}$ are the heat capacities of the pure components in the phase $P$. These heat capacities are known from pure components' measurements. Within the temperature range of interest, they are very well approximated by Taylor series of the second order in the temperature:

$$
\begin{aligned}
c_{p, i}^{P}(T)= & c_{p, i, \mathrm{ref}}^{P}+\left(\frac{\mathrm{d} c_{p, i}^{P}}{\mathrm{~d} T}\right)_{T_{\mathrm{ref}}}\left(T-T_{\mathrm{ref}}\right) \\
& +\frac{1}{2}\left(\frac{\mathrm{d}^{2} c_{p, i}^{P}}{\mathrm{~d} T^{2}}\right)_{T_{\mathrm{ref}}}\left(T-T_{\mathrm{ref}}\right)^{2}
\end{aligned}
$$

where $c_{p, i, \text { ref }}^{P}$ is the pure component heat capacity in phase $P$ at some reference temperature $T_{\text {ref }}$, and $\frac{\mathrm{d} c_{p, i}^{P}}{\mathrm{~d} T}, \frac{d^{2} c_{p, i}^{P}}{\mathrm{~d} T^{2}}$ are the first and the second derivatives at $T_{\text {ref }}$.

The cooling power of the calorimeter as a function of temperature within the one-phase regions can be calculated straightforwardly from:

$I_{\text {cool }}^{\text {exp }}=\left(c_{p, \text { ves }}+c_{p, \text { mix }}^{P}\right) \frac{\mathrm{d} T}{\mathrm{~d} t}$.

The heat is withdrawn from the system by radiation to the adiabatic shields and by conduction through the wire heater. In accordance with this, theoretically the cooling power can be expressed as the sum of a radiation and a conduction term:

$$
\begin{aligned}
I_{\text {cool }}^{\text {theor }} & =I_{\text {radiation }}+I_{\text {conduction }} \\
& =q_{1}\left(T_{\text {system }}^{4}-\left(T_{\text {system }}-\Delta T\right)^{4}\right)+\left(q_{2}+q_{3} T_{\text {system }}\right)
\end{aligned}
$$

where $\Delta T$ is a temperature difference between the system and the inner adiabatic shield, being set to $10 \mathrm{~K}$ during the cooling experiments.

By fitting the experimental cooling power (Eq. (4)) to the theoretical expression (Eq. (5)), parameters $q_{1}, q_{2}$ and $q_{3}$ are obtained for each measured mixture. Basically, these parameters should not depend on the content of the vessel. Nevertheless, to rule out the effects of small fluctuations in the experimental conditions we determined cooling parameters for each cooling experiment. The obtained values are given in Table 1. The resulting cooling powers as a function of temperature are shown in Fig. 2, illustrating that, despite the differences in the parameters, they are quite close as we expected. 
Table 1

Cooling power parameters for each mixture cooled in the adiabatic calorimeter

\begin{tabular}{|c|c|c|c|c|}
\hline Mixture & $x_{2}$ & $q_{1} \times 10^{11}\left(\mathrm{~J} \mathrm{~K}^{-1} \mathrm{~s}^{-1}\right)$ & $q_{2} \times 10^{3}\left(\mathrm{~J} \mathrm{~s}^{-1}\right)$ & $q_{3} \times 10^{5}\left(\mathrm{~J} \mathrm{~K}^{-1} \mathrm{~s}^{-1}\right)$ \\
\hline I & 0.2937 & 1.3372 & -2.1740 & 2.8007 \\
\hline II & 0.4791 & 1.3938 & -1.4711 & 2.4425 \\
\hline III & 0.5312 & 1.3878 & -1.9066 & 2.6865 \\
\hline IV & 0.5338 & 1.2658 & -3.9556 & 3.5783 \\
\hline $\mathrm{V}$ & 0.6025 & 1.3712 & -1.7688 & 2.6282 \\
\hline VI & 0.7976 & 1.3423 & -2.0353 & 2.7332 \\
\hline
\end{tabular}

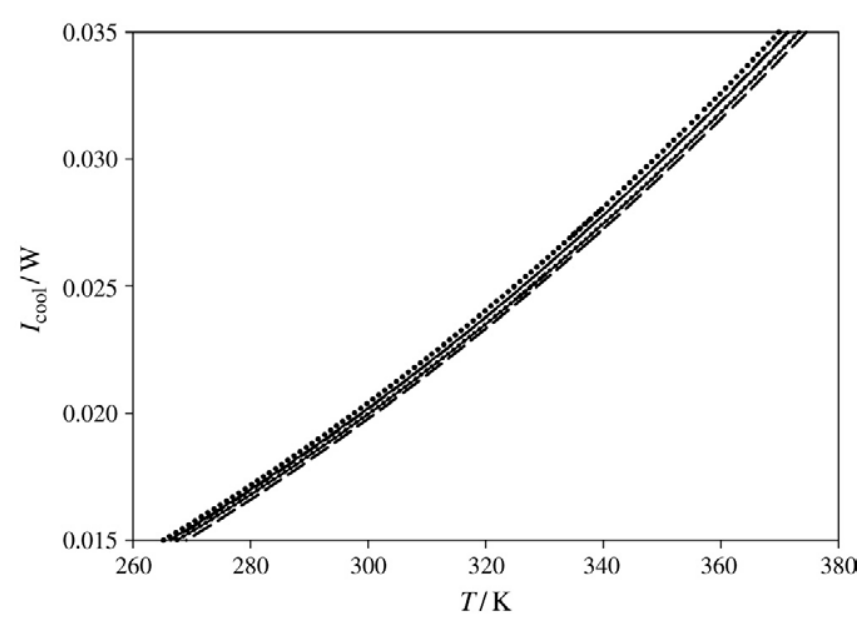

Fig. 2. The cooling power of the adiabatic calorimeter $\left(I_{\mathrm{cool}}\right)$ as a function of temperature, calculated for each investigated mixture by Eq. (5) using parameters from Table 1.

Once the cooling power is defined as a function of the system's temperature, the enthalpy of the mixture can be calculated as a function of temperature for the applied cooling by:

$$
\begin{aligned}
H_{\mathrm{mix}}^{\exp }(T)= & H_{\mathrm{mix}}^{\exp }\left(T_{\text {ref }}\right)+H_{\mathrm{ves}}\left(T_{\text {ref }}\right)-H_{\mathrm{ves}}(T) \\
& +\int_{T_{\text {ref }}}^{T} I_{\mathrm{cool}}\left(\frac{\mathrm{d} T^{\prime}}{\mathrm{d} t}\right)^{-1} \mathrm{~d} T^{\prime}
\end{aligned}
$$

where the integration constant, $H_{\mathrm{ves}}\left(T_{\mathrm{ref}}\right)$, is chosen such that the enthalpy of the liquid mixture is equal to zero at $T_{\text {ref }}$, which is chosen to be $365 \mathrm{~K}$.

\section{Qualitative interpretation of the measured enthalpy path}

For each composition of the mixture, the enthalpy path during the described cooling is calculated by Eq. (6) and plotted in Fig. 3. Note that all enthalpy curves contain two kinks, which are typical for the crystallization process as opposed to the presented melting enthalpy curves.

Upon continuous cooling of the liquid phase, the first kink in the enthalpy curves appears at point 0 and marks the onset of crystallization by nucleation. Once the crystallization process starts, the solid phase evolves rapidly as is evident from the registered temperature increase between points 0 and 1 . In all cases the nucleation temperature $T_{0}$ is found to be about $8 \mathrm{~K}$ lower than the corresponding liquidus temperature of the mixture of the given composition $z$. At $T_{0}$ the liquid phase is highly supersaturated. This means that during the initial

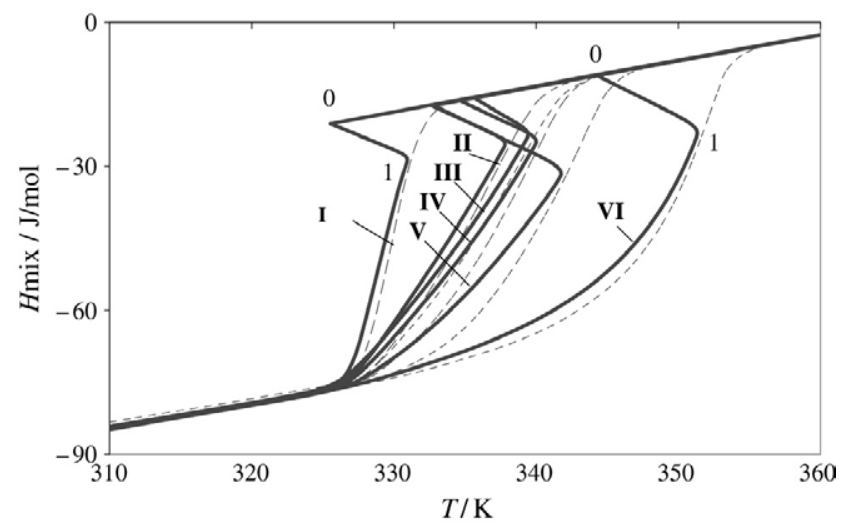

Fig. 3. The enthalpy change of mixtures of different compositions $\left(H_{\text {mix }}\right)$ with temperature $(T)$, obtained from heating (dashed line) and cooling (solid line) the mixtures in the adiabatic calorimeter. Denotations for different compositions are in line with those in Table 1 . Points 0 and 1 indicate kinks on the cooling enthalpy paths (shown here for only two mixtures for clarity of the figure).

crystallization the composition of the growing solid phase is not necessarily lying on the solidus line of the equilibrium phase diagram, but its determination would require the knowledge of the so-called kinetic phase diagram, that follows from a non-equilibrium approach, such as the one given in Ref. [15]. Thus, for the amount of solid phase formed along the path 0-1 the segregation may deviate from the equilibrium segregation. In contrast, once being at $T_{1}$ we assume that equilibrium is established between the remaining liquid phase and the surface of the solid phase. This assumption is supported by the fact that further growth of the solid phase from $T_{1}$ downward is accompanied by gradual temperature decrease, which points to significantly slower growth of the solid phase than in the initial stage. Accordingly, the solidification front, i.e. the solid phase that is growing at the surface, is assumed to be in (near) equilibrium with the remaining liquid phase.

Such a description of solidification implies that the solid phase grows in layers of different compositions, where each layer is in equilibrium with the remaining liquid phase at the given temperature during cooling. This approach considers a definite time span at the given temperature as opposed to the equilibrium, which assumes an infinite time span and thus allows the equilibrium between completely homogeneous phases. This picture of the crystallization of a solid solution is known in the literature as the shell model [16].

The above qualitative description and assumptions regarding the experimental cooling enthalpy curves give base to our quantitative modeling of the crystallization process. 


\section{Quantitative analysis, kinetic model}

According to the previous discussion, the impact of kinetics is significant so that a successful description of the crystallization process requires both thermodynamic and kinetic factors to be taken into account. We introduce a kinetic way of modeling the crystallization process by deriving the expressions for calculating the enthalpy path for a given set of excess parameters. These parameters determine the excess contribution to the thermodynamic properties of the mixture, and quantify the degree of miscibility of the components in the given phase. A given excess quantity for a phase $P$ is commonly expressed as a polynomial function of the composition. Here we adopt the Redlich-Kister expansion [17], reading:

$A^{P, \operatorname{exc}}=x(1-x) \sum_{n=0}^{N} a_{n}^{P, \operatorname{exc}}(1-2 x)^{n}$

where $x=x_{2}$ is the mole fraction of component 2, which is usually chosen to be the component with the highest melting temperature, and $A$ stands for the excess enthalpy, entropy or the free Gibbs energy obeying:

$G_{\mathrm{mix}}^{P, \mathrm{exc}}=H_{\mathrm{mix}}^{P, \text { exc }}-T S_{\operatorname{mix}}^{P, \text { exc }}$.

During the phase transition in the adiabatic calorimeter, the enthalpy change of the system consists of a contribution for

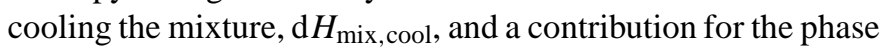
transformation, $\mathrm{d} H_{\text {trans. }}$. So we can write:

$\mathrm{d} H_{\text {mix }}=\mathrm{d} H_{\text {mix }, \text { cool }}+\mathrm{d} H_{\text {trans }}=c_{p, \text { mix }} \mathrm{d} T+\Delta H_{\text {fus }} \mathrm{d} s$

where $\Delta H_{\text {fus }}$ is the composition dependent enthalpy of fusion of the mixture and $\mathrm{d} s$ refers to the amount of the solid phase that is formed within the time interval $\mathrm{d} t$ corresponding to the temperature change $\mathrm{d} T$.

Let us turn now to the qualitative analysis of the experimental data, where the two parts of the crystallization process were distinguished. In the initial part the growth of the solid phase is fast and segregation may deviate from the equilibrium segregation, while during the second part the crystallization proceeds slowly and we assume that the solid phase at the surface is in (near) equilibrium with the existing liquid phase. To start, we first derive expressions for the evolution of solid fraction and the enthalpy as a function of temperature during the second part of the crystallization, i.e. starting from $T_{1}$. Here we refer to Fig. 4, where the cooling path for the melt of overall composition $z$ is schematically presented in the phase diagram from Ref. [4]. As diffusion in the solid phase is neglected, the lever rule, which is based on complete equilibrium, is not valid, but we can still apply a differential form of the lever rule. This implies that the amount of solid formed between $T$ and $T-\Delta T$ is given by:

$\Delta s=(1-s) \frac{x_{\mathrm{eq}}^{\operatorname{liq}}(T)-x_{\mathrm{eq}}^{\operatorname{liq}}(T-\Delta T)}{x_{\mathrm{eq}}^{\mathrm{sol}}(T-\Delta T)-x_{\mathrm{eq}}^{\operatorname{liq}}(T-\Delta T)}$

where $x_{\mathrm{eq}}^{\text {liq }}$ and $x_{\mathrm{eq}}^{\mathrm{sol}}$ are the equilibrium mole fractions (of component 2) of the liquid and the solid phase at the

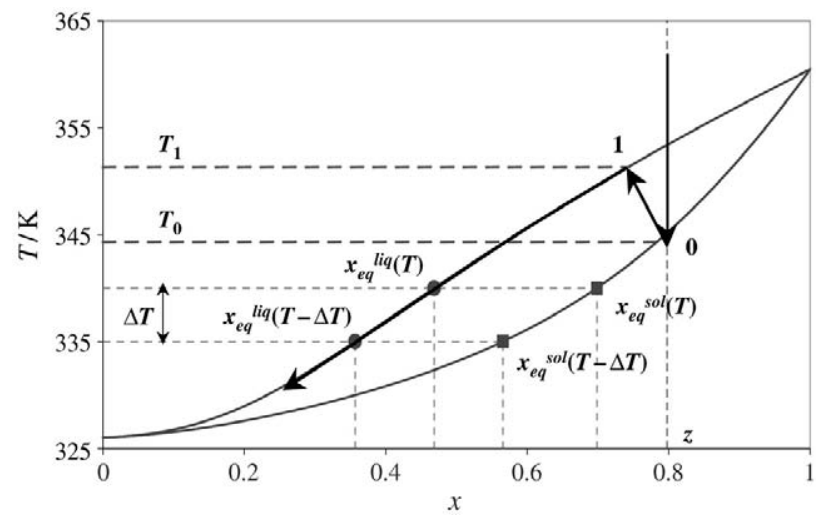

Fig. 4. The crystallization path for the mixture of overall composition $z$, schematically presented in the liquid-solid phase diagram from Ref. [4]. Upon continuous cooling, the nucleation of solid phase occurs at point $0\left(T_{0}\right)$, from where temperature rises until point $1\left(T_{1}\right)$ and then starts decreasing again. For a description of solid fraction evolution below $T_{1}$ we use Eq. (10), where during temperature decrease $\Delta T$ the liquid phase will change composition along the liquidus line, from $x_{\mathrm{eq}}(T)$ to $x_{\mathrm{eq}}(T-\Delta T)$, while the composition of the growing solid moves from $x_{\mathrm{eq}}^{\mathrm{sol}}(T)$ to $x_{\mathrm{eq}}^{\mathrm{sol}}(T-\Delta T)$.

corresponding temperature. For $\Delta T \rightarrow 0$, Eq. (10) becomes:

$\mathrm{d} s=-(1-s) \frac{\mathrm{d} x_{\mathrm{eq}}^{\mathrm{liq}} / \mathrm{d} T^{\prime}}{x_{\mathrm{eq}}^{\mathrm{sol}}\left(T^{\prime}\right)-x_{\mathrm{eq}}^{\operatorname{liq}}\left(T^{\prime}\right)} \mathrm{d} T^{\prime}$

implying:

$\int_{s_{0}}^{s} \frac{\mathrm{d} s}{1-s}=-\int_{T_{1}}^{T} \frac{\mathrm{d} x_{\mathrm{eq}}^{\mathrm{liq}} / \mathrm{d} T^{\prime}}{x_{\mathrm{eq}}^{\mathrm{sol}}\left(T^{\prime}\right)-x_{\mathrm{eq}}^{\operatorname{liq}}\left(T^{\prime}\right)} \mathrm{d} T^{\prime}$.

Finally, by analytic integration of Eq. (12) we find that the solid phase fraction as a function of temperature from $T_{1}$ downward is given by:

$s(T)=1-\left(1-s_{0}\right) \exp \left(\int_{T_{1}}^{T} \frac{\mathrm{d} x_{\mathrm{eq}}^{\mathrm{liq}} / \mathrm{d} T^{\prime}}{x_{\mathrm{eq}}^{\mathrm{sol}}\left(T^{\prime}\right)-x_{\mathrm{eq}}^{\mathrm{liq}}\left(T^{\prime}\right)} \mathrm{d} T^{\prime}\right)$.

Similarly, the theoretical enthalpy path is found by substitution of Eq. (11) into the energy balance Eq. (9), and integrating, which leads to:

$$
\begin{aligned}
H_{\mathrm{mix}}^{\mathrm{theor}}(T)= & \int_{T_{1}}^{T}\left(c_{p, \text { mix }}-(1-s(T))\right. \\
& \left.\times \frac{\mathrm{d} x_{\mathrm{eq}}^{\mathrm{liq}} / \mathrm{d} T^{\prime}}{x_{\mathrm{eq}}^{\mathrm{sol}}\left(T^{\prime}\right)-x_{\mathrm{eq}}^{\operatorname{liq}}\left(T^{\prime}\right)} \Delta H_{\mathrm{fus}}\right) \mathrm{d} T^{\prime}
\end{aligned}
$$

where $\Delta H_{\text {fus }}$ is the composition dependent enthalpy of fusion given by:

$$
\Delta H_{\mathrm{fus}}=\left(1-x_{\mathrm{eq}}^{\mathrm{sol}}\right) \Delta H_{\mathrm{fus}, 1}+x_{\mathrm{eq}}^{\mathrm{sol}} \Delta H_{\mathrm{fus}, 2}+\Delta H^{\mathrm{exc}}\left(x_{\mathrm{eq}}^{\mathrm{sol}}\right) \text {. }
$$

Here $\Delta H_{\text {fus, } 1}$ and $\Delta H_{\text {fus, } 2}$ stand for the temperature dependent enthalpies of melting of the pure components, while $\Delta H^{\mathrm{exc}}$ is the difference between the excess enthalpy in the liquid and solid phase. Our calculated enthalpy paths from $T_{1}$ downward are obtained by numerical integration of Eq. (14), inserting the 
temperature dependent solid fraction of Eq. (13). However, in order to perform this integration, the amount of solid being formed between $T_{0}$ and $T_{1}, s_{0}$, and its average composition, $x_{\mathrm{av}}^{\mathrm{sol}}$, have to be determined.

Although we assume that the surface of the solid at $T_{1}$ is of equilibrium composition, the total initial solid phase, $s_{0}$, is in fact inhomogeneous. However, its average composition $x_{\mathrm{av}}^{\mathrm{sol}}=x_{2, \mathrm{av}}^{\mathrm{sol}}$ must satisfy the mass balance equation:

$\left(1-s_{0}\right) x_{\mathrm{eq}}^{\mathrm{liq}}+s_{0} x_{\mathrm{av}}^{\mathrm{sol}}=z$.

As the surface of the solid phase is in equilibrium with the remaining liquid at $T_{1}$, the following relation holds for both components of the binary mixture:

$\gamma_{i, \text { eq }}^{\text {liq }} x_{i, \text { eq }}^{\text {liq }}=x_{i, \text { eq }}^{\text {sol }} \gamma_{i, \text { eq }}^{\text {sol }} \exp \left(-\frac{\Delta H_{i, \text { fus }}-T_{1} \Delta S_{i, \text { fus }}}{R T_{1}}\right)$

for $i=1,2$, where $\Delta S_{i, \text { fus }}$ is the temperature dependent melting entropy of the pure component $i$. The excess property of the mixture in phase $P=\mathrm{liq}$, sol is expressed in the terms of activity coefficient of component $i$ in that phase, $\gamma_{i, \text { eq }}^{P}$, which is related to the excess free Gibbs energy of the phase, $G_{\operatorname{mix}}^{P, \text { exc }}$, by:

$R T \ln \left(\gamma_{i, \text { eq }}^{P}\right)=\frac{\partial G_{\operatorname{mix}}^{P, \text { exc }}}{\partial N_{i}^{P}}$

where $N_{i}^{P}$ is the amount of component $i$ in phase $P$.

From the experiment the value of the enthalpy of the mixture at $T_{1}$ is known and should be equal to:

$H_{\text {mix }}^{\text {theor }}\left(T_{1}\right)=\left(1-s_{0}\right) H^{\text {liq }}+s_{0} H^{\text {sol }}$

where $H^{\text {liq }}$ and $H^{\text {sol }}$ are the liquid and solid enthalpies at $T_{1}$, given by:

$H^{\mathrm{liq}}=\left(1-x_{\mathrm{eq}}^{\mathrm{liq}}\right) H_{1}^{* \text { liq }}+x_{\mathrm{eq}}^{\text {liq }} H_{2}^{* \text {,liq }}+H^{\text {liq,exc }}\left(x_{\mathrm{eq}}^{\mathrm{liq}}\right)$

$H^{\mathrm{sol}}=\left(1-x_{\mathrm{av}}^{\mathrm{sol}}\right) H_{1}^{*, \mathrm{sol}}+x_{\mathrm{av}}^{\mathrm{sol}} H_{2}^{*, \text { sol }}+H^{\mathrm{sol}, \mathrm{exc}}\left(x_{\mathrm{av}}^{\mathrm{sol}}\right)$

with $H_{i}^{*, P}$ being the pure component enthalpies at $T_{1}$ and $H^{P \text {,exc }}$ the excess enthalpies in phase $P=$ liq, sol. The liquid phase is not treated as completely ideal, since we adopted the excess properties of the liquid phase as determined in Ref. [4].

With the coupled equations of form Eq. (17) for both components, the mass balance Eq. (16) and the enthalpy balance at $T_{1}$ (Eqs. (19)-(21)), the four unknowns $\left(s_{0}, x_{\mathrm{eq}}^{\mathrm{liq}}, x_{\mathrm{eq}}^{\mathrm{sol}}, x_{\mathrm{av}}^{\mathrm{sol}}\right)$ can be determined.

In Table 2 the experimental temperatures $T_{0}$ and $T_{1}$ are given for each mixture, together with the calculated initial solid fractions $s_{0}$ and relevant compositions for the initial part of the crystallization.

To illustrate the performance of the proposed kinetic model, the calculated cooling enthalpy path is compared with the experimental enthalpy path for the mixture of composition $z=0.7976$, assuming the excess properties to be as determined by van der Linde [4]. In Fig. 5 we also include the enthalpy path as calculated from the model, which assumes complete equilibrium at any time during the crystallization process. Clearly, much better agreement between the experimental and
Table 2

Nucleation and equilibrium temperatures $T_{0}$ and $T_{1}$ for each investigated composition of the mixture, and corresponding results from modeling the initial crystallization: initial solid fraction $s_{0}$, equilibrium compositions of liquid and solid phase at $T_{1}\left(x_{\text {liq }}^{\mathrm{eq}}\right.$ and $\left.x_{\text {sol }}^{\mathrm{eq}}\right)$ and average composition of the initial solid $\left(x_{\mathrm{sol}}^{\mathrm{av}}\right)$

\begin{tabular}{lllllll}
\hline$x_{2}$ & $T_{0} / K$ & $T_{1} / K$ & $s_{0}$ & $x_{\text {liq }}^{\mathrm{eq}}$ & $x_{\text {sol }}^{\mathrm{eq}}$ & $x_{\text {sol }}^{\mathrm{av}}$ \\
\hline 0.2937 & 325.537 & 330.932 & 0.1826 & 0.2262 & 0.3529 & 0.5958 \\
0.4791 & 332.641 & 337.808 & 0.1892 & 0.4338 & 0.6498 & 0.6729 \\
0.5312 & 334.646 & 339.998 & 0.1962 & 0.4613 & 0.6772 & 0.8157 \\
0.5338 & 335.622 & 339.435 & 0.1624 & 0.4472 & 0.6670 & 0.9809 \\
0.6025 & 332.811 & 341.751 & 0.3294 & 0.5187 & 0.7322 & 0.7731 \\
0.7976 & 344.306 & 351.329 & 0.2746 & 0.7461 & 0.8898 & 0.9335 \\
\hline
\end{tabular}

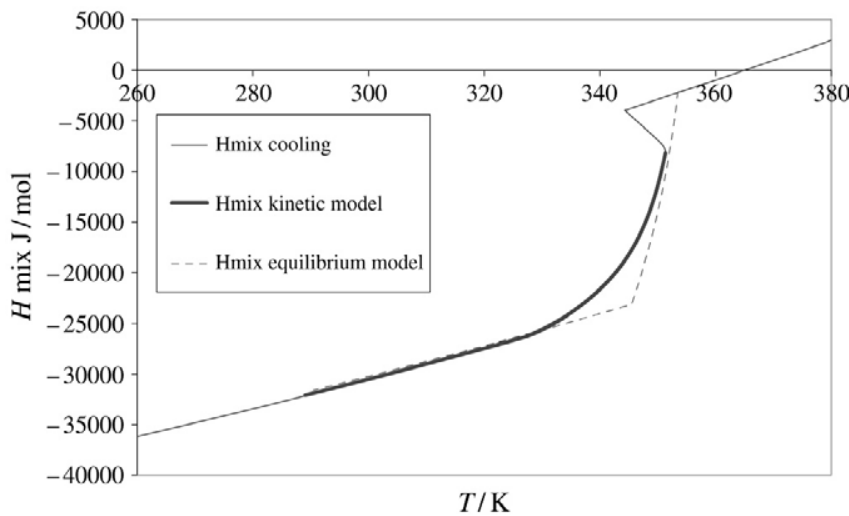

Fig. 5. The experimental enthalpy cooling path of the mixture $\left(H_{\text {mix }}\right.$ cooling, solid line) of overall composition $z=0.7976$, compared to those reproduced from the kinetic ( $H_{\text {mix }}$ kinetic model, solid bold line) and equilibrium model ( $H_{\text {mix }}$ equilibrium model, dashed line). All three enthalpy paths are calculated by using the excess properties of the mixture as determined in Ref. [4].

kinetic enthalpy curves affirms the validity of the kinetic modeling, while the equilibrium approach fails in describing properly the crystallization process.

\section{Determination of excess parameters}

As has been demonstrated, the kinetic model successfully describes the crystallization process when the excess properties of the phases are known. On the other hand, the kinetic model can be used to determine the excess properties by fitting the theoretical enthalpy path (Eq. (14)) to the experimental one as measured in the adiabatic calorimeter during cooling. In principle, the difference between the theoretical and experimental value of the enthalpy at the given temperature $T_{i}$, starting from $T_{1}$ downward, is minimized:

$F=\sum\left(H_{\mathrm{mix}, i}^{\text {theor }}-H_{\mathrm{mix}, i}^{\exp }\right)^{2}$.

The result of the proposed procedure is a set of the excess parameters that define dimensionless excess properties, written as follows:

$\frac{\Delta H_{\mathrm{sol}}^{\mathrm{liq}, E}}{R T}=x(1-x)\left(h_{21}(1-x)+h_{12} x\right)=x(1-x) \tilde{h}^{\mathrm{exc}}$ 
Table 3

Excess parameters that determine dimensionless excess functions (excess enthalpy, excess entropy and excess free Gibbs energy)

\begin{tabular}{llllllll}
\hline$x_{2}$ & $h_{12}$ & $h_{21}$ & $s_{12}$ & $g_{12}$ & $g_{21}$ & $h_{\text {exc }}$ & $g_{\text {exc }}$ \\
\hline 0.2937 & 0.611 & 0.741 & 0.213 & 0.399 & 0.529 & 0.597 & 0.699 \\
0.4791 & 0.688 & 0.604 & 0.073 & 0.615 & 0.531 & 0.616 & 0.574 \\
0.5312 & 0.757 & 0.755 & 0.270 & 0.487 & 0.485 & 0.715 & 0.489 \\
0.5338 & 0.723 & 0.799 & 0.264 & 0.459 & 0.535 & 0.574 & 0.531 \\
0.6025 & 0.753 & 0.569 & 0.151 & 0.602 & 0.419 & 0.649 & 0.531 \\
0.7976 & 0.753 & 0.524 & 0.230 & 0.523 & 0.294 & 0.625 & 0.488 \\
\hline
\end{tabular}

$\frac{\Delta S_{\mathrm{sol}}^{\mathrm{liq}, E}}{R}=x(1-x)\left(s_{21}(1-x)+s_{12} x\right)=x(1-x) \tilde{s}^{\operatorname{exc}}$

$\frac{\Delta G_{\mathrm{sol}}^{\mathrm{liq}, E}}{R T}=x(1-x)\left(g_{21}(1-x)+g_{12} x\right)=x(1-x) \tilde{g}^{\mathrm{exc}}$.

Note that the relevant quantity for the phase behavior is the difference in the excess free energy for the liquid and solid phase. During the calculation, we restrict ourselves to fitting with three parameters, two for the excess enthalpy $\left(h_{12} \neq h_{21}\right)$ and one for the excess entropy $\left(s_{12}=s_{21}\right)$, while the parameters that describe the excess Gibbs energy follow from the above relation Eq. (8). As we observe no excess heat capacity, the excess properties are not dependent on temperature, but only on the composition of the mixture. For each mixture of different composition that has been measured in the adiabatic calorimeter, the set of the excess parameters is obtained and given in Table 3. Calculated excess functions of composition $\tilde{h}^{\text {exc }}, \tilde{s}^{\text {exc }}$ and $\tilde{g}^{\text {exc }}$ are shown in Fig. 6. Their values can be fitted by a polynomial of an optional order, which will imply different numbers of the relevant Redlich-Kister excess parameters. The proposed procedure provides both excess enthalpy and entropy as functions of composition. These two quantities give the excess free Gibbs energy, enabling the calculation of the phase diagram. Accordingly, the excess enthalpy and entropy curves presented in Fig. 6 are fitted in the linear functions of composition, so that finally two Redlich-Kister parameters are obtained for these excess properties, being: $\Delta H_{\mathrm{sol}, 0}^{\mathrm{liq}, E}=$ $2146.64 \mathrm{~J} \mathrm{~mol}^{-1}, \Delta H_{\mathrm{sol}, 1}^{\text {liq }, E}=-26.1 \mathrm{~J} \mathrm{~mol}^{-1} ; \Delta S_{\mathrm{sol}, 0}^{\text {liq, },}=$ $1.14 \mathrm{~J}(\mathrm{~K} \mathrm{~mol})^{-1}, \Delta S_{\text {sol, } 1}^{\text {liq, }}=-0.252 \mathrm{~J}(\mathrm{~K} \mathrm{~mol})^{-1}$. Using these values the phase diagram is calculated and compared to the phase diagram as determined in Ref. [4] (see Fig. 7). Similarity between the presented phase diagrams illustrates that the crystallization follows closely the equilibrium phase diagram, as expected since the crystallization is performed very slowly.

\section{Summary}

The validity of the introduced kinetic model is demonstrated by successful reproduction of the enthalpy path of the mixture during cooling for the known excess properties. The applicability of the model is extended so that it yields the excess properties when the cooling path of the mixture is at disposal. By fitting the measured enthalpy of the mixture to

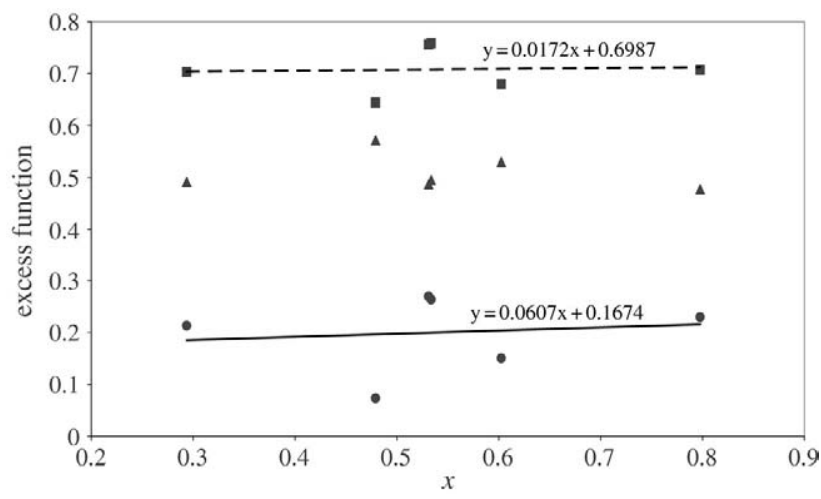

Fig. 6. Dimensionless excess quantities as functions of overall composition: $\mathbf{\square}$ - excess enthalpy $\tilde{h}^{\text {exc }} ; \boldsymbol{O}-$ excess entropy $\tilde{s}^{\text {exc }} ; \boldsymbol{\Delta}$ - excess Gibbs energy $\tilde{g}^{\text {exc }}$.

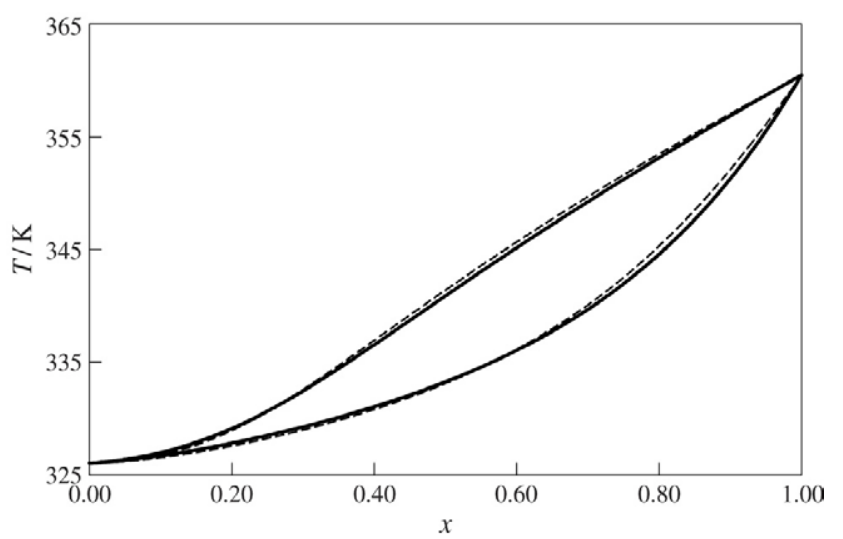

Fig. 7. The phase diagrams calculated using the excess properties from equilibrium model in Ref. [4] (dashed line) and from the kinetic model (solid line).

the theoretical expression, the parameters that define the excess enthalpy and entropy of the solid phase as the functions of overall composition, are calculated. In this way, the excess quantities can be obtained by using a relatively simple method, which basically requires only the knowledge of the cooling curve of the mixture. Finally, the phase diagram is achieved having the advantage over traditionally determined phase diagrams, in the sense that both excess enthalpy and entropy are derived without adopting the approach of complete equilibrium between totally homogeneous phases.

\section{References}

[1] A.N. Campbell, L.A. Prodan, An apparatus for refined thermal analysis exemplified by a study of the system p-dichlorobenzene-pdibromobenzene-p-chlorobromobenzene, J. Amer. Chem. Soc. 70 (1948) 553.

[2] M.T. Calvet, M.A. Cuevas-Diarte, Y. Haget, P.R. van der Linde, H.A.J. Oonk, Binary p-dihalobenzene systems - correlation of thermochemical and phase-diagram data, CALPHAD 15 (3) (1991) 225.

[3] R. Stosch, S. Bauerecker, H.K. Cammenga, The fusion behaviour of mixed crystals. A comparison between experimental and calculated calorimetric curves, Z. Phys. Chem. 194 (1996) 231.

[4] P.R. van der Linde, Molecular mixed crystals from thermodynamic point of view, Ph.D. Thesis, Utrecht University, 1992.

[5] J.A. Bouwstra, Thermodynamic and structural investigations of binary systems, Ph.D. Thesis, Utrecht University, 1985. 
[6] A.C.G. van Genderen, C.G. de Kruif, H.A.J. Oonk, Properties of mixed crystalline organic material prepared by zone leveling, Z. Phys. Chem. Neue Folge 107 (1977) 167.

[7] Z. Zbioski, Zone growth: a simple procedure for growing organic crystals from the melt or from the vapour phase, J. Cryst. Growth 58 (1982) 335.

[8] P.A. Reynolds, Disorder in the crystal structures of paradichlorobenzene, Mol. Phys. 29 (2) (1975) 519.

[9] M.A. Korshunov, Distribution of component molecules in pdibromobenzene-p-dichlorobenzene solid solutions from Raman effect data, Crystallo. Rep. 48 (3) (2003).

[10] J.H. Los, M. Matovic, Effective kinetic phase diagrams, J. Phys. Chem. B 30 (2005) 14632.

[11] M. Matovic, J.C. van Miltenburg, J.H. Los, Metastability in solid solution growth, J. Cryst. Growth 275 (2005) e211-e217.
[12] J.H. Los, W.J.P. van Enckevort, E. Vlieg, E. Flöter, Metastable states in multicomponent liquid-solid systems I: a kinetic crystallization model, J. Phys. Chem. B (2002) 106.

[13] J.C. van Miltenburg, H.A.J. Oonk, Heat capacities and derived thermodynamic functions of 1-Octadecanol, 1-Nonadecanol, 1-Eicosanol and 1-Docosanol between $10 \mathrm{~K}$ and 370 K, J. Chem. Eng. Data 46 (2001) 90-97.

[14] J.C. van Miltenburg, A.C.G. van Genderen, G.J.K. van den Berg, Design improvements in adiabatic calorimetry - the heat capacity of cholesterol between 10 and 425 K, Thermochim. Acta 383 (1998) 13-19.

[15] Z. Chvoj, J. Šesták, A. Tøiska, Kinetic Phase Diagrams, Elsevier, Amsterdam, 1991.

[16] R.E. Timms, Prog. Lipid Res. 23 (1984) 1.

[17] O. Redlich, A.T. Kister, Ind. Eng. Chem. 40 (1948) 345. 